\title{
Techniques, indications and complications of pericardiocentesis
}

\author{
Mohammed Shafik Elkahwagy ${ }^{1 *}$, Ahmad Faraj Alenezi ${ }^{2}$, Hibah Farhan Alenezi ${ }^{3}$, \\ Bader Naji Al-Hussain ${ }^{2}$, Mohammad Ahmad Allwaim ${ }^{4}$, Almukhtar Mohammed Almomatten ${ }^{5}$, \\ Mohammed Ali Alkhuwaysah', Mohammed Abdullah Alwabari', \\ Thumamah Mahmoud Alarabi ${ }^{7}$, Mohammed Hamed Albarakati ${ }^{8}$, \\ Naif Abdullah Albadrani', Nasser Dakhel Alrehaili ${ }^{10}$
}

\author{
${ }^{1}$ Department of Cardiothoracic Surgery, King Fahad General Hospital, Jeddah, Saudi Arabia \\ ${ }^{2}$ College of Medicine, Arabian Gulf University, Manama, Bahrain \\ ${ }^{3}$ College of Medicine, Northern Border University, Arar, Saudi Arabia \\ ${ }^{4}$ College of Medicine, Medical University of Lodz, Lodz, Poland \\ ${ }^{5}$ College of Medicine, King Faisal University, Alhofuf, Saudi Arabia \\ ${ }^{6}$ College of Medicine, Wroclaw Medical University, Wroclaw, Poland \\ ${ }^{7}$ Department of Family Medicine, Primary Healthcare, Medina, Saudi Arabia \\ ${ }^{8}$ College of Medicine, Gdansk Medical University, Gdansk, Poland \\ ${ }^{9}$ Unaizah College of Medicine, Qassim University, Unaizah, Saudi Arabia \\ ${ }^{10}$ College of Medicine, King Abdulaziz University, Rabigh, Saudi Arabia
}

Received: 28 August 2021

Accepted: 05 October 2021

*Correspondence:

Dr. Mohammed Shafik Elkahwagy,

E-mail: noora_rahimuddinn@hotmail.com

Copyright: (c) the author(s), publisher and licensee Medip Academy. This is an open-access article distributed under the terms of the Creative Commons Attribution Non-Commercial License, which permits unrestricted non-commercial use, distribution, and reproduction in any medium, provided the original work is properly cited.

\begin{abstract}
The main aim of pericardiocentesis is to remove excess fluid in the pericardial space and enhance the functions of the heart to intervene against the development of many complications. Accordingly, the procedure is usually performed to manage cardiac tamponade to correct secondary hypotension that attributes to the low stroke volume as a result of the external pressure of the pericardial fluid on the chambers of the heart. In the present literature review, we have discussed the techniques, indications, and complications of pericardiocentesis. Furthermore, the main techniques and approaches include computed tomography-guided pericardiocentesis, echo-guided, and fluoroscopy-guided techniques. These modalities can be successfully used for hemodynamically unstable patients to adequately remove excess fluid to normalize ventricular filling and maintain adequate cardiac output to the peripheral tissues. Considerations for using the modality have been made for small effusions and many authors suggest that it should not be routinely used in these situations. Many major and minor complications can occur secondary to conducting pericardiocentesis. The major complications might include lacerations of the intercostal vessels of the coronary arteries, injury to the cardiac chambers, death, puncture of the peritoneal cavity or abdominal viscera, pneumopericardium, pneumothorax which might require chest intubation, pericardial decompression syndrome, and ventricular arrhythmias. Therefore, carefully approaching these patients and deciding the best management plan, in addition to providing proper interventional approaches for the potential development of these complications is essential to enhance the intended outcomes and enhance the quality of care.
\end{abstract}

Keywords: Pericardiocentesis, Complications, Indications, Cardiac tamponade 


\section{INTRODUCTION}

Cardiac tamponade is life-threatening and is a timesensitive condition that needs to be promptly detected and managed. ${ }^{1}$ The diagnosis of the condition is usually based on the clinical features of the affected patients. However, with recent advances in medical field, echocardiography aided much in the diagnostic outcomes of cardiac tamponad. $^{2}$ Moreover, many management approaches have been introduced and reported among various studies in literature for management of cardiac tamponade. Among these, pericardiocentesis has been validated as a safe and feasible approach that can effectively manage these cases and enhance associated outcomes.

Furthermore, the main aim of pericardiocentesis is to remove excess fluid in the pericardial space and enhance the functions of the heart to intervene against the development of many complications. ${ }^{3,4}$ Accordingly, the procedure is usually performed to manage cardiac tamponade to correct secondary hypotension that attributes to the low stroke volume as a result of the external pressure of the pericardial fluid on the chambers of the heart. The procedure can be life-saving for many patients, like when cardiothoracic surgery cannot be conducted and in cases of type A aortic aneurism dissection. ${ }^{5-7}$ In the present review, we aim to discuss the techniques, indications, and complications of pericardiocentesis, based on evidence obtained from the current investigations in the literature.

\section{METHODS}

This literature review is based on an extensive literature search in Medline, Cochrane, and EMBASE databases which was performed on $20^{\text {th }}$ August 2021 using the medical subject headings $(\mathrm{MeSH})$ or a combination of all possible related terms. This was followed by the manual search for papers in Google Scholar while the reference lists of the initially included papers. Papers discussing the techniques, indications, and complications of pericardiocentesis were screened for relevant information, with no limitations on date, language, age of participants, or publication type.

\section{DISUCSSION}

\section{Techniques}

Many approaches have been validated in the literature to successfully conduct pericardiocentesis. These include computed tomography-guided pericardiocentesis, echoguided, and fluoroscopy-guided techniques. In 1979, the echo-guided pericardiocentesis technique was first reported in the literature as a simple and safe approach that is now being widely used in different clinical settings. Using echocardiography will allow the performing physicians to adequately locate the ideal needle trajectory and entry site, in addition to evaluating the size and location of the effusion. ${ }^{8}$ Moreover, evidence also shows that the modality has two different approaches. The first approach is the echo-guided approach, by which echocardiography is continuously used to help with the process of needle introduction and fluid aspiration. On the other hand, the other approach mainly uses echocardiography for assistance to localize the needle site of entry, and after this, the physician memorizes the site of entry with no further use of echocardiography, and this approach was the one that was first reported by the Mayo clinic. Another recent report also suggested that to successfully introduce the needle for successful aspiration of fluid from the pericardium, a needle carrier that is mounted on the ultrasound device is advisable to achieve such outcomes., ${ }^{9,10}$ In another context, the fluoroscopy-guided approach was the first reported modality for conducting pericardiocentesis. The approach is performed using a needle that contains a contrast medium that is inserted in the subxiphoid approach, at which the needle is inserted at $30^{\circ}$ towards the left shoulder. The contrast agent medium is used to confirm that the needle is placed within the targeted space. Besides, it has been demonstrated that it is important to use at least two angiographic projections to make sure that the approach is correctly done and the needle has been successfully inserted within the intended space. ${ }^{11}$ Although the approach is feasible, it poses a potential risk to the physician and the patient of being subjected to exposure. Besides, it can only be performed in heart catheterization laboratories. This approach is recommended in cases of iatrogenic tamponade. The computed tomography-guided technique was also validated among studies in the literature. By using this approach, physicians can effectively evaluate the size and extension of the effusion and mark the entrance point of the aspiration needle. Besides, computed tomography is then furtherly used to indicate that needle was successfully inserted in the intended position. It can also be used for detection of any underlying comorbidity or etiology within chest. However, no continuous visualization of needle can be obtained, and the patient is subjected to an increased risk of radiation exposure. Moreover, modality is time-consuming, not logistically available, and not widely feasible..$^{8,12,13}$ The puncture site has previously been validated among the different studies and is being critically discussed elsewhere..$^{9,10,14}$

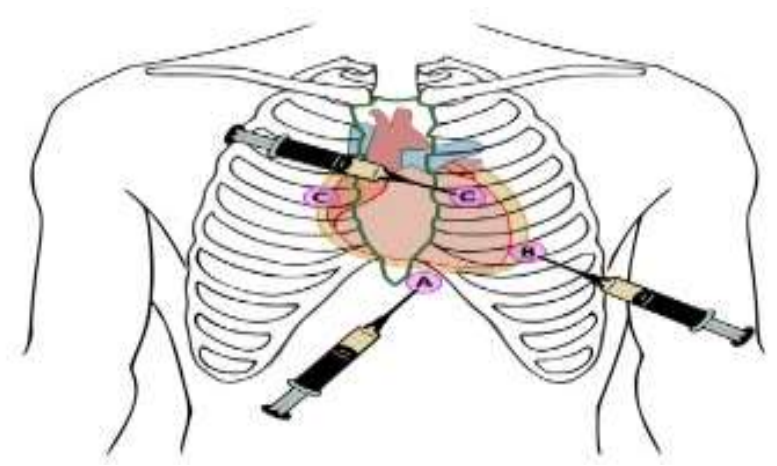

Figure 1: Puncture sites for pericardiocentesis approach. $^{15}$ 


\section{Indications}

Pericardiocentesis is usually recommended as an urgent procedure for hemodynamically unstable patients to adequately remove excess fluid to normalize ventricular filling and maintain adequate cardiac output to the peripheral tissues. ${ }^{3,9,10}$ On the other hand, in cases when the affected patient is hemodynamically stable, the procedure can be successfully performed within hours after presentation, which can adequately enable the attending physicians to carefully plan for the procedure to obtain better outcomes. Besides, using a scoring system has been proposed among studies in the literature as a validated approach to decide which patients should be urgently indicated for pericardiocentesis and which can wait for some hours in cases of cardiac tamponade. The components of this scoring system mainly include three categories, upon which patients can be classified based on the urgency of their status. These components include clinical presentation, etiology, and findings on echocardiography. ${ }^{16,17}$ Furthermore, studies have indicated that for patients that do not suffer from hemodynamics compromise, urgent pericardiocentesis can be delayed. However, the approach has been indicated for cases with symptomatic manifestations, moderate-large effusions, and in cases when there is an absent or minimal response to the medical management modalities. $^{3,18-20}$ Besides, it has been argued that pericardiocentesis should also be indicated for patients with small effusions that develop secondary to tuberculous or bacterial infections, and neoplasm-related pericarditis. Moreover, large $(>20 \mathrm{~mm}$ in diameter based on an echocardiographic assessment), and chronic (that lasted for $>3$ months) pericarditis is also an indicator for performing pericardiocentesis. In another context, using pericardiocentesis is not recommended for diagnostic purposes in cases of mild-moderate effusions for several reasons. These include-1) idiopathic or viral pericarditis is usually self-limiting and resolves spontaneously, and the treatment is usually based on the administration of anti-inflammatory drugs, 2) the limited diagnostic efficacy of pericardiocentesis in these situations because the underlying etiology is usually detected by other noninvasive approaches, and 3) the high risk of developing complications and related morbidities which is even higher than the benefits that might potentially be obtained from the diagnostic value of the procedure. ${ }^{21,22}$ It should be noted that evidence in the literature indicates that performing pericardiocentesis is not associated with absolute contraindications, despite the potential development of shock or cardiac tamponade. However, it has been demonstrated that post-infarction rupture of the free wall and aortic dissection are contraindications against performing surgical tamponade (or needle pericardiocentesis). This is attributable to the huge risk that might result in the myocardial ruptured wall and dissected aorta secondary to the restoration of the systemic blood pressure and the rapid increase in the pericardial decompression events. On the other hand, it has been demonstrated that drainage of small amounts of the excess fluid and pericardiocentesis can be performed in cases when the patient is unstable, and the surgical management could not be rapidly conducted to maintain normal levels of systemic blood pressure (usually around $90 \mathrm{mmHg}$ ) until the surgery is indicated and performed. ${ }^{1}$ Moreover, anticoagulation therapy, uncorrected coagulopathy, and thrombocytopenia (platelet count $<50,000 \mathrm{~mm}^{3}$ ) are relative contraindications for performing pericardiocentesis. ${ }^{23,24}$

\section{Complications}

Many studies have assessed the prevalence of complications among patients that underwent pericardiocentesis. Among the big studies that reported the prevalence rate, a rate of $0.3-3.9 \%$, and $0.4-20 \%$ for major and minor complications, respectively, following either fluoroscopic or echo-guided pericardiocentesis..$^{9,25-}$ ${ }^{27}$ Besides, among the major complications, many events might be life-threatening and others can induce serious morbidities. Some of the most serious complications might include lacerations of the intercostal vessels or the coronary arteries, injury to the cardiac chambers, death, puncture of the peritoneal cavity or abdominal viscera, pneumopericardium, pneumothorax which might require chest intubation, pericardial decompression syndrome, and ventricular arrhythmias. The development of coronary or myocardial puncture might present as an asymptomatic event and be discovered late with the development of intrapericardial thrombus or hemopericardium. It has been estimated that pericardial compression is usually a rare complication following pericardiocentesis. However, it has been observed that the complication is a serious one that might end up with mortality, especially because the event might be characteristically associated with a wide range of scenarios, including cardiogenic shock, and pulmonary edema. It was also estimated that this complication usually develops following the process of pericardiocentesis by a few hours-days. However, it has been demonstrated the actual mechanism for the development of this event is not adequately comprehended based on evidence from the current studies in the literature. On the other hand, some theories exist. For instance, it has been explained that resistant peripheral vasoconstriction that is related to catecholamines administration might significantly lead to an increase in the pre-load of the right side, which then can induce a significant overload on the left ventricle. No apparent guidelines have been reported for the management or prevention of this complication. However, the only recommended action is to adequately remove excess fluid to normalize the systemic and central venous blood pressure within the first few hours after the complication develops. ${ }^{16}$ Furthermore, many minor or non-serious complications were also reported among the current studies in the literature. These might include supraventricular arrhythmias, transient vasovagal bradycardia and hypotension, pleuropericardial fistulas, and pneumothorax with no development of hemodynamic 
instability. Besides, it has been suggested among the various studies that special attention should be given to anticoagulant therapy during the periprocedural management of the complications and during pericardiocentesis. Furtherly, it has been demonstrated that pericardiocentesis should be delayed when the patient suffers from many coagulation disorders until the patient is provided with adequate management modalities for these disorders or was introduced to adequate blood transfusion frequencies. Besides, it is widely known that full anticoagulation practices might pose a risk factor for the development of complications and might have a serious risk on tamponade in cases of patients suffering from iatrogenic-related pericardial effusion. ${ }^{16}$ Pericardiocentesis is usually performed as a life-saving approach in cases when there is a significant hemodynamic instability and cardiac tamponade, which indicates that the procedure should be urgently performed to enhance the prognosis in such cases. It should be noted that all of the aforementioned findings are based on observational studies only with no evidence obtained from randomized controlled trials, which or absent in the literature. Following the administration of pericardiocentesis, it has been indicated that the prognosis of the underlying cases is significantly dependant on the presence of comorbidity and the underlying disease that priorly caused the development of the cardiac tamponade. For instance, the presence of neoplasms is an indicator of a poor prognosis while in cases of viral infections or idiopathic etiologies, it has been reported that the prognosis is usually excellent in such cases. Pericardiocentesis might also be indicated as a diagnostic approach to enhance the management plan and reduce the development of restrictive pericarditis. Therefore, it has been recommended that the modality should be mandatorily conducted in cases of neoplastic, tuberculous, or bacterial pericarditis to successfully perform a fluid analysis and establish a proper diagnosis. Furthermore, it has been estimated that the prognosis of chronic massive pericarditis and associated effusion is good in general, however, an estimated rate of $35 \%$ for the risk of developing tamponade has been reported. ${ }^{28,29}$

\section{CONCLUSION}

The main techniques and approaches in pericardiocentesis include computed tomography-guided pericardiocentesis, echo-guided, and fluoroscopy-guided techniques. These modalities can be successfully used for hemodynamically unstable patients to adequately remove excess fluid to normalize ventricular filling and maintain adequate cardiac output to the peripheral tissues. Considerations for using the modality have been made for small effusions and many authors suggest that it should not be routinely used in these situations. Many major and minor complications can occur secondary to conducting pericardiocentesis. Therefore, carefully approaching these patients and deciding the best management plan, in addition to providing proper interventional approaches for the potential development of these complications is essential to enhance the intended outcomes and enhance the quality of care.

Funding: No funding sources

Conflict of interest: None declared

Ethical approval: Not required

\section{REFERENCES}

1. Cruz I, Stuart B, Caldeira D. Controlled pericardiocentesis in patients with cardiac tamponade complicating aortic dissection: experience of a centre without cardiothoracic surgery. Euro heart J Acute cardiovascular care. 2015;4(2):124-8.

2. Akyuz S, Zengin A, Arugaslan E. Echo-guided pericardiocentesis in patients with clinically significant pericardial effusion. Outcomes over a 10year period. Herz. 2015;40(2):153-9.

3. Adler Y, Charron P, Imazio M. 2015 ESC Guidelines for the diagnosis and management of pericardial diseases: The Task Force for the Diagnosis and Management of Pericardial Diseases of the European Society of Cardiology (ESC)Endorsed by: The European Association for Cardio-Thoracic Surgery (EACTS). Eur Heart J. 2015;36(42):2921-64.

4. Krikorian JG, Hancock EW. Pericardiocentesis. Am j med. 1978;65(5):808-14.

5. Chetrit M, Lipes J, Mardigyan V. A Practical Approach to Pericardiocentesis With Periprocedural Use of Ultrasound Training Initiative. Canadian $\mathrm{j}$ cardiol. 2018;34(9):1229-32.

6. Schusler R, Meyerson SL. Pericardial Disease Associated with Malignancy. Curr cardiol rep. 2018;20(10):92.

7. Vilela EM, Ruivo C, Guerreiro CE. Computed tomography-guided pericardiocentesis: a systematic review concerning contemporary evidence and future perspectives. Therap advances cardiovascular dis. 2018;12(11):299-307.

8. Jung HO. Pericardial effusion and pericardiocentesis: role of echocardiography. Korean circulation j. 2012;42(11):725-34.

9. Maggiolini S, Gentile G, Farina A. Safety, Efficacy, and Complications of Pericardiocentesis by RealTime Echo-Monitored Procedure. Am J Cardiol. 2016;117(8):1369-74.

10. Tsang TSM, Freeman WK, Sinak LJ, Seward JB. Echocardiographically Guided Pericardiocentesis: Evolution and State-of-the-Art Technique. Mayo Clin proceedings. 1998;73(7):647-52.

11. Kumar R, Sinha A, Lin MJ. Complications of pericardiocentesis: A clinical synopsis. Int J Crit Illn Inj Sci. 2015;5(3):206-12.

12. Neves D, Silva G, Morais G. Computed tomographyguided pericardiocentesis-A single-center experience. Revista Portuguesa de Cardiologia. 2016;35(5):285-90.

13. Maggiolini S, De Carlini CC, Ferri LA. The role of early contrast-enhanced chest computed tomography in the aetiological diagnosis of patients presenting 
with cardiac tamponade or large pericardial effusion.

Eur Heart J- Cardiovascular Imaging. 2015;17(4):421-8.

14. Tsang TSM, Seward JB. Pericardiocentesis Under Echocardiographic Guidance. Eur J Echocardiography. 2001;2(1):68-9.

15. Sinnaeve PR, Adriaenssens T. A contemporary look at pericardiocentesis. Trends cardiovascular med. 2019;29(7):375-83.

16. Ristić AD, Imazio M, Adler Y. Triage strategy for urgent management of cardiac tamponade: a position statement of the European Society of Cardiology Working Group on Myocardial and Pericardial Diseases. Eur Heart J. 2014;35(34):2279-84.

17. Pham TS, Reda A, Ngan Nguyen TT. Blood exchange transfusion in viral hepatitis in a small infant: a case report. Transfusion and apheresis sci. 2020;59(6):102907.

18. Cooper CA. Centesis studies in critical care. Critcare nursing clin North Am. 2010;22(1):95-108.

19. Thomas F, Clemmer TP, Orme JF, Jr. A survey of advanced trauma life support procedures being performed by physicians and nurses used on hospital aeromedical evacuation services. Aviation, space environmental med. 1985;56(12):1213-5.

20. Alpert EA, Amit U, Guranda L, Mahagna R, Grossman SA, Bentancur A. Emergency department point-of-care ultrasonography improves time to pericardiocentesis for clinically significant effusions. Clin exp emergency med. 2017;4(3):128-32.

21. Maggiolini S, Osculati G, Vitale G. Utility and safety of diagnostic pericardiocentesis. Eur Heart J. 2005;26(10):1046-7.

22. Son PT, Reda A, Viet DC. Exchange transfusion in the management of critical pertussis in young infants: a case series. Vox Sang. 2021.
23. Lekhakul A, Fenstad ER, Assawakawintip C. Incidence and Management of Hemopericardium: Impact of Changing Trends in Invasive Cardiology. Mayo Clin proceedings. 2018;93(8):1086-95.

24. Honasoge AP, Dubbs SB. Rapid Fire: Pericardial Effusion and Tamponade. Emergency med clin North Am. 2018;36(3):557-65.

25. Tsang TSM, Enriquez-Sarano M, Freeman WK. Consecutive $1127 \quad$ Therapeutic Echocardiographically Guided Pericardiocenteses: Clinical Profile, Practice Patterns, and Outcomes Spanning 21 Years. Mayo Clin proceedings. 2002;77(5):429-36.

26. Akyuz S, Zengin A, Arugaslan E. Echo-guided pericardiocentesis in patients with clinically significant pericardial effusion. Herz. 2015;40(2):153-9.

27. Thieu H, Bach Dat B, Nam NH. Antibiotic resistance of Helicobacter pylori infection in a children's hospital in Vietnam: prevalence and associated factors. Minerva med. 2020;111(5):498-501.

28. Narula J, Choudhury A, Sharma A. Pericardiocentesis can be nasty. Accidents do occur while "Rail-roading" Sheaths and pigtails! Ann cardiac anaesthesia. 2018;21(3):290-92.

29. Lekhakul A, Assawakawintip C, Fenstad ER, et al. Safety and Outcome of Percutaneous Drainage of Pericardial Effusions in Patients with Cancer. Am j cardiol. 2018;122(6):1091-4.

Cite this article as: Elkahwagy MS, Alenezi AF, Alenezi HF, Al-Hussain BN, Allwaim MA, Almomatten AM et al. Techniques, indications and complications of pericardiocentesis. Int J Community Med Public Health 2021;8:5540-4. 